\title{
Forecasting of Electricity Demand by Hybrid ANN-PSO Models
}

\author{
Atul Anand, Anna University, Chennai, India
}

L. Suganthi, Anna University, Chennai, India

\begin{abstract}
Developing economies need to invest in energy projects. Because the gestation period of the electric projects is high, it is of paramount importance to accurately forecast the energy requirements. In the present paper, the future energy demand of the state of Tamil Nadu in India, is forecasted using an artificial neural network (ANN) optimized by particle swarm optimization (PSO) and by Genetic Algorithm (GA). Hybrid ANN Models have the potential to provide forecasts that perform well compared to the more traditional modelling approaches. The forecasted results obtained using the hybrid ANN-PSO models are compared with those of the ARIMA, hybrid ANN-GA, ANN-BP and linear models. Both PSO and GA have been developed in linear and quadratic forms and the hybrid ANN models have been applied to five-time series. Amongst all the hybrid ANN models, ANN-PSO models are the best fit models in all the time series based on RMSE and MAPE.
\end{abstract}

\section{KEYWORDS}

Artificial Neural Network, Energy Demand, Forecasting, Particle Swarm Optimization, Tamil Nadu, Tamil Nadu Generation and Distribution Company Limited

\section{INTRODUCTION}

In order to ensure sustained growth for developing economies such as the state of Tamil Nadu in India, long term planning of energy resources is imperative to bridge the electrical energy gap. The allocation of capital resources requires an accurate model for forecasting electricity demand that can enable the optimal utilization of scarce resources. It is also useful for resource planning and for attracting investments in the field of energy. In recent reports on the energy policies of India, simple measures of GDP-elasticity and energy intensities have been used for demand forecasting for ten or more years (Government of India,2006). In this paper, the total electric energy demand is segregated into agriculture, residential, commercial, and industrial sectors. For each sector, hybrid artificial neural network (hybrid ANN) models have been developed using the state of art optimizing techniques, namely, particle swarm optimization (PSO) and Genetic Algorithm (GA). Hybrid ANN models have the potential to provide forecasts that perform well, compared to the more traditional modeling approaches. For each optimizing technique, both linear and quadratic forms have been developed. The four hybrid ANN models that are compared in this paper are: -ANN-PSO(Linear), ANN-PSO(Quadratic), ANN-GA(Linear) and ANN-GA(Quadratic). The results of each sector are compared with linear, autoregressive integrated moving average (ARIMA), and artificial neural network with backward propagation (ANN-BP) models. The best fit model for each sector is selected based on error indices such as root mean square error (RMSE) and mean absolute percentage error (MAPE).

\section{DOI: 10.4018/IJEOE.2017100105}

This article published as an Open Access article distributed under the terms of the Creative Commons Attribution License (http://creativecommons.org/licenses/by/4.0/) which permits unrestricted use, distribution, and production in any medium, provided the author of the original work and original publication source are properly credited. 
The study of the electric energy demand profile of the State of Tamil Nadu in Figure 1 shows a non-linear growth with undulations superimposed on the profile. For such a non-linear profile, in the past, many applications used the traditional statistical models, such as the autoregressive model, moving average model and the auto regressive moving average. These models perform well when the data lie within the range of past observations but they perform poorly while predicting extremes and also when the data lies near the limits. Moreover, conventional optimization might either fail to obtain a feasible solution or be trapped in local optima. However, with the advent of ANN and inspired by its strong ability of non-linear mapping, it has been applied to the field of energy forecasting. ANN does not require to specify a particular model form. Rather, the model is adaptively formed based on the features presented from the data. This data driven approach is suited for many empirical data sets where no theoretical guidance is available to suggest an appropriate data generating process. Therefore, ANN has been considered in the present study. Various independent socio-economic factors were considered to develop a forecasting model, as Table 1 shows, and Pearson correlations were found. On this basis, the per capita energy index, number of consumers, and consumer price index were taken as independent variables.

For non-linear demand profile, hybrid ANN models are designed to obtain near-optimal objective function values after optimization. The heuristic techniques such as GA and PSO are considered promising alternatives in this type of problem. They have been proved to be robust and powerful tools for many kinds of optimization problems (Unler, 2008). The detailed procedure for the hybrid ANN models which are proposed in the study is as follows:

- In the first step, PSO and GA optimization techniques are applied to the sectors of total electric demand, namely, agriculture, residential, commercial and industries sectors based on socioeconomic indicators. PSO and GA optimization techniques are applied separately in linear and quadratic forms using the data from the year 1991 to 2000 for each time series. The optimizing techniques (PSO and GA) are used to train ANN which projects the demand from year 2001 to

Figure 1. Demand profile in the period 1991-2015

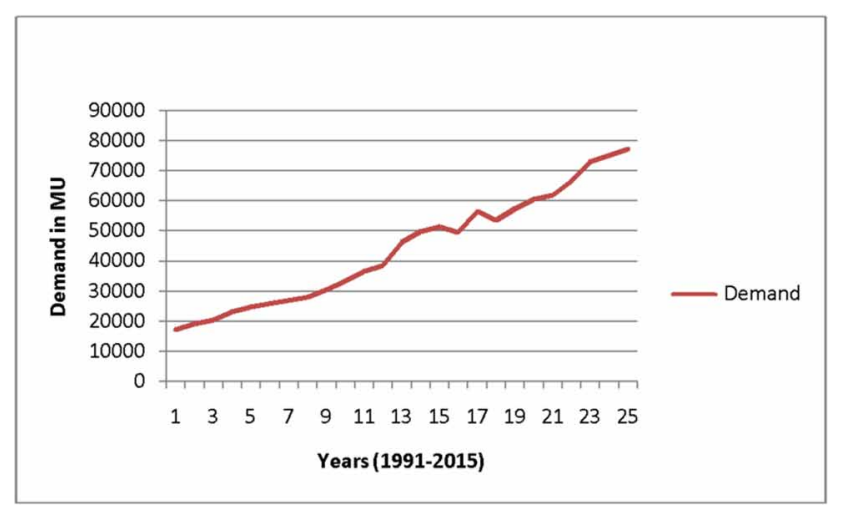

Table 1. Details of correlations among socio-economic factors

\begin{tabular}{|c|c|c|c|c|c|c|}
\hline $\begin{array}{c}\text { Pearson } \\
\text { Correlations }\end{array}$ & $\begin{array}{c}\text { Per Capita } \\
\text { Energy Index }\end{array}$ & Consumers & Population & $\begin{array}{c}\text { Consumer } \\
\text { Price Index }\end{array}$ & $\begin{array}{c}\text { Gross State } \\
\text { Domestic } \\
\text { Product }\end{array}$ & $\begin{array}{c}\text { Per } \\
\text { Capita } \\
\text { Income }\end{array}$ \\
\hline $\begin{array}{c}\text { Total Electricity } \\
\text { Demand }\end{array}$ & $\mathbf{0 . 6 8}$ & $\mathbf{0 . 7 6}$ & $\mathbf{0 . 6 5}$ & $\mathbf{0 . 8}$ & $\mathbf{- 0 . 1 8 6}$ & $\mathbf{- 0 . 0 8 2}$ \\
\hline
\end{tabular}


2015. A comparison is made between the actual values and forecasted values of the electricity demand using ANN-PSO-Linear, ANN-PSO-Quadratic, ANN-GA, ANN-GA-Quadratic, ANNBP, ARIMA and linear models. The performances of various models are compared and the best fit model is selected based on RMSE and MAPE;

- In the second step, total electric energy demand is forecasted based on the best fit model up to the year 2020. This study uses the technique mentioned in Fienberg and Genethlion's (2005) work using SCILAB. The coding has been done for the inertial weight model of PSO. IBM SPSS 2.0 software is used for running ANN.

The paper is divided into following sections, namely, Literature review, Forecasting Techniques, Determination of weight coefficients, Discussions, and Conclusions.

\section{LITERATURE REVIEW}

A review of the demand forecasting approaches suggests the existence of a large variety of techniques used by different sets of users. Bhattacharyya and Timilsina (2009) suggested that models can be categorized into two broad categories: simple approaches and sophisticated approaches. Simple models rely on simple indicators commonly used for forecasting such as growth rates, elasticities (especially income elasticity), specific or unit consumption and energy intensity. Westoby and Pearce (1984) noted that most of the work on energy forecasting used the "energy ratio" (which is popularly known as "energy intensity") and the "energy coefficient" (i.e. the elasticity of the energy demand with respect to the national income or GDP). Similarly, Codoni, Park and Ramani (1985) reported the use of the income elasticity of demand for an energy assessment study of Korea. Grover and Chandra (2006) report that Indian state agencies rely on income elasticities for forecasting primary energy and electricity demand. Harvey (1997) proposed an alternative method called the structural time series models, which have been applied to the energy demand, among others settings, by Hunt, Judge, and Ninomiya (2003). These studies have generally focused on the aggregated demand and considered variables such as GDP and price, but they do not capture the technological changes. The first systematic elaboration of the method and an application in France were reported by Chateau and Lapillonne (1978). Worrel (2004) argued in favour of disaggregation of the total energy demand into relevant homogenous end-use categories (Government of India -2006). Dahl (1994a) suggested that although models are found to test the per capita energy and total energy consumption, aggregation can cause heteroscedasticity when the population varies across the sample. Ibrahim (1985) reviewed the energy demand forecasting efforts in Arab countries using time series, single-equation models, and the aggregated approach noting that none of them meet the requirements of policy analysis. Similarly, Chern and Soberon-Ferrer (1986) analysed the structural changes in energy demand in developing countries. Ishiguro and Akiyama (1995) analysed the energy demand in five Asian countries, namely China, India, South Korea, Thailand and Indonesia both at the aggregate level and the sector level using a simple econometric model and provided forecasts for these countries up to 2005 . Pesaran M.H, Smith R, Akiyama T (1998) conducted a major study that analyzed the energy demand in 11 Asian developing countries using an autoregressive distributed lag model for co-integration both at the aggregate and sector levels. Shiwei Yu and Yi-Ming Wei (2012) developed a PSO-GA optimal model to estimate primary energy demand of China. Unler Alper (2008) highlighted the use of PSO in linear and quadratic form for forecasting energy demand. Araby and Yorino (2010) showed the utility of hybrid PSO technique for electricity markets. Zhu and Wang (2011) developed a hybrid model with adaptive particle swarm optimization algorithm for electricity demand forecasting for China. 


\section{FORECASTING TECHNIQUES}

\section{The ARIMA Model}

ARIMA is a times series model which is based on the assumption that the data possesses an internal structure such as trend analysis, auto-correlation or seasonality. The time series model predicts future values based on previously observed values. ARIMA methods assume that the time series are generated from linear processes. Linear models have advantages in that they can be understood and analyzed in great details and are easy to explain. However, they may be totally inappropriate if the underlying mechanism is non-linear. In fact, most real world systems are often non-linear (Granger \& Terasvirta (1993). In this study, the ARIMA $(1,0,1)$ model has been developed for each sub sector.

\section{The Hybrid ANN-PSO Model}

ANNs have been widely used in demand forecasting since 1990 (Kodeeswararamanathan et al., 2014). ANN is well suited for problems where the solutions require knowledge of factors whose relationships are difficult to specify (Kaastra \& Boyd, 1996). According to Zhang et al. (1998) in such problems, good theoretical guesses of the underlying laws governing the systems are difficult to fathom (Guoqiang Zhang et al., 1998). The functional value in an ANN model can be written as:

$$
y=f\left(x_{1}, x_{2}, \ldots x_{p}\right)
$$

where $x_{1}, x_{2}, \ldots, x_{p}$ are the ' $\mathrm{p}$ ' independent variables and $\mathrm{y}$ are is the independent variable:

$$
y_{t+1}=f\left(y_{t}, y_{t-1}, \ldots, y_{t-p}\right)
$$

where $y_{t}$ is the observation of time " $\mathrm{t}$ ".

ANN has been optimized with PSO technique. Particle swarm optimization (PSO) method, first published by Kennedy and Eberhart in 1995 (Westoby \& Pearce, 1984), is based on a population of points that are at first stochastically deployed on a search field. A variant of the PSO method was developed by Shi and Eberhart in 1998 (Codoni et al., 1985) in which a modification of the speed equation improves the convergence by inserting a time dependent variable in the following equations:

$$
v_{t+1}=v_{t}+R_{1} * C_{1} *\left(g-x_{t}\right)+R_{2} * C_{2} *\left(p-x_{t}\right)
$$

$$
x_{t+1}=x_{t}+v_{t+1}
$$

where $C 1$ and $C 2$ are knowledge factors, $R 1$ and $R 2$ are random numbers, $g$ is the location of the leader, $p$ the personal best location, $v_{t}$ is the velocity at iteration " $\mathrm{t}$ " and $x_{t}$ is the position at iteration "t". PSO searches for the most fitted members in the search space by minimizing the error.

\section{Normalization of Data}

Raw data is not normally used directly in process modeling of ANN. This is due to the difference in magnitude of the process variables. The data was scaled to prevent data with larger magnitude from overriding the smaller and impede the premature learning process. For normalization of the raw data 
the year 1991 was taken as the base year and its parameters were used for comparing the raw data of the subsequent years as follows:

$$
v_{t}^{\text {norm }}=\frac{V_{t}}{V_{\text {Base }}}
$$

where $\mathrm{t}$ is the year $v_{t}^{\text {norm }}$ is the normalized reading for the $\mathrm{t}^{\text {th }}$ year and $v_{\text {Base }}$ is the reading of the year 1991 taken as base year.

The forecasted electricity demand, y (Linear) in ANN-PSO (Linear) is given as follows:

$$
y(\text { linear })=\sum_{1}^{n} X_{i}(t) * W_{i}(t)
$$

where $X_{i}(t)$ is the normalized value of the $i^{t h}$ socio economic factor and $W_{i}(\mathrm{t})$ is corresponding weight. $n$ is the total number of socioeconomic factors.

\section{Hybrid ANN-PSO-Quadratic Model (QANN-P)}

The weights of the input variables are calculated as per the Equation 3. The Equations 1 and 2 represents the generalized PSO model but in Quadratic PSO model the quadratic terms are introduced to the second and third terms in Equation 1 and the evolution equations become:

$$
v_{t+1}=v_{t}+R_{1} * C_{1} * \operatorname{sign}\left(g-x_{t}\right) *\left(g-x_{t}\right)^{\wedge} 2+R_{2} * C_{2} * \operatorname{sign}\left(p-x_{t}\right) *\left(p-x_{t}\right)^{\wedge} 2
$$

$$
x_{t+1}=x_{t}+v_{t+1}
$$

Therefore, the Quadratic PSO algorithm based on the evolution Equations 4 and 5 satisfies the requirements for describing the swarm intelligence behavior of bird flocking. The Quadratic PSO algorithm has the ability to simulate swarm intelligence of bird flocking and its difference with the standard PSO is in the introduction of the quadratic terms in the evolution equation. It improves the diversity of the swarm so that higher performance in global optimization. Quadratic PSO projects the input variables for the years 2001 to 2015 while using the data from 1991 to 2000 as input. Following equation is used for forecasting the demand using QANN-P:

$$
\begin{aligned}
y(\text { Quadratic }) & =X_{1}^{2}(t) * W_{1}(t)+X_{2}^{2}(t) * W_{2}(t)+X_{3}^{2}(t) * W_{3}(t) \\
& +W_{12}(t) * X_{1}(t) * X_{2}(t)+W_{23}(t) * X_{2}(t) * X_{3}(t)+W_{13}(t) * X_{1}(t) * X_{3}(t)
\end{aligned}
$$

where $X_{i}(t)$ is the normalized value of the $i^{t h}$ socio economic indicator, $W_{i}(t)$ is corresponding weight for the $t^{t h}$ year of the $i^{t h}$ indicator and $W_{i j}(t)$ is corresponding weight between the $i^{t h}$ and $j^{\text {th }}$ indicators. 


\section{Hybrid ANN-GA Model (ANN-GA)}

The performance of weights evolution using GA depends on the number of populations and generations. If these parameters were set too low, the evolution may converge to immature solution. However, the larger number of populations and generations would require longer computation time for convergence (Araby \& Yorino, 2010). In order to find the weights of the input variables GA was run on SCILAB as per the parameters shown in Table 2, for the following linear equation for forecasted demand " $y$ " during the " $\mathrm{t}$ " the iteration is as follows:

$$
y(t)=\sum_{1}^{n} W_{i}(t) * X_{i}(t)
$$

where $W_{i}$ is the weight of the $i^{t h}$ parameter, $X_{i}$ is the $i^{\text {th }}$ socioeconomic factor and $n$ is the total number of factors.

\section{ANN-GA-Quadratic Model (QANN-GA)}

Another version of Hybrid ANN-GA Model has been developed with the quadratic equation as follows:

$$
y(t)=\sum_{1}^{n} X_{i}^{2} * W_{i}
$$

where $y(t)$, is the Energy Demand for the year ' $\mathrm{t}$ ', $W_{i}$ represents the normalized weights, $W_{1}, W_{2}, W_{3}$ of PerCapita Energy Density $\left(X_{1}\right)$, Consumer Price Index $\left(X_{2}\right)$ and Number of Consumers $\left(X_{3}\right)$ respectively.

Using the normalized data for the inputs the weights are calculated using GA with parameters as shown in Table 3. The weights are calculated using GA subject to the condition that the values of normalized value of output (n-GA-Q) is as close as possible to the normalized value of the actual demand (nDemand). The weights and the normal input values are fed as nodes in the Artificial Neural Network.

\begin{tabular}{|r|r|r|}
\hline S.no & Parameters of GA & Values \\
\hline 1 & Population Size & 100 \\
\hline 2 & Probability of mutation & 0.1 \\
\hline 3 & Probalility of cross mutation & 0.7 \\
\hline 4 & Number of couples & 110 \\
\hline 5 & Number of generations & 10 \\
\hline
\end{tabular}


Table 3. Parameters of PSO

\begin{tabular}{|c|c|c|}
\hline S. No & Parameters & Value \\
\hline 1. & Number of Population & 50 \\
\hline 2. & Maximum Number of Iterations $\left(\right.$ iter $\left._{\max }\right)$ & 200 \\
\hline 3. & Initial Inertia $\left(w_{\max }\right)$ & 0.8 \\
\hline 4. & Final Inertia $\left(w_{\text {min }}\right)$ & 0.2 \\
\hline 5. & Personal Best Knowledge Factor $\left(c_{1}\right)$ & 2 \\
\hline 6. & Global Best Knowledge Factor $\left(c_{2}\right)$ & 2 \\
\hline
\end{tabular}

\section{DETERMINATION OF WEIGHT COEFFICIENTS}

In each hybrid, ANN-PSO approach each neural network (NN) defines the attributes of position and velocity. The position is related to weight of neural network. The velocity refers to updating of ANN's weights. The function of PSO in ANN is to get the best set of weights (particle position) where several particles are trying to move to get best solution. For neural network implementation, the fitness value corresponds to a forward propagation through the network and position vector of the network. The particle's best neighbor and global best particle are used to guide the particle new solutions. In the end the global best particle's position serves as the answer. Hence the function of PSO in ANN is to get the best set of weights. The iterative approach of PSO followed in the study is as follows:

Step 1: Initialize a population size, positions and velocities of agents, and the number of weights and biases. As shown in Table 3 the number of population is taken as 50 .

Step 2: The current best fitness achieved by particle $p$ is set as pbest. The pbest with best value is set as gbest and this value is stored. The personal best knowledge factor has been taken as 2 .

Step 3: Evaluate the desired optimization fitness function $F(x)$ for each particle as follows:

$$
F(x)=\sqrt{\sum_{j=1}^{m}\left(E_{\text {actual }}-E_{\text {predicted }}\right)^{2}}
$$

where $E_{\text {actual }}$ and $E_{\text {predicted }}$ are the actual and predicted values of the electric energy demand respectively, $\mathrm{m}$ is the number of observations. The fitness function is formulated such that after each iteration, some new set of values are obtained that are added to the existing values, and a new set of values are obtained. The energy demand values that have minimal $\mathrm{F}(\mathrm{x})$ are taken for the next iteration of the PSO. The present study uses the 'Inertial Weight Model' developed by Shi and Eberhart with the 'Radius Improvement Model' developed by Salmon (Araby \& Yorino, 2010). 
Step 4: Compare the evaluated fitness value $F(p)$ for each particle, p, with its pbest value. If $F(p)<$ pbest then pbest $=F(p)$ and bestxp $=x p, x p$ represents the current coordinates of particle $p$, and bestxp represents the coordinates corresponding to particle $p$ 's best fitness so far.

Step 5: The objective function value is calculated for new positions of each particle. If a better position is achieved by a particle, pbest value is replaced by the current value. As in Step 1, gbest value is selected among pbest values. If the new gbest value is better than previous gbest value, the gbest value is replaced by the current gbest value and this value is stored. if $f p<$ gbest then $g b e s t$ $=p$, where gbest is the particle having the overall best fitness over all particles in the swarm.

Step 6: Change the velocity and location of the particle according to Equation 1 and Equation 2.

Step 7: If the maximum number of a predetermined iterations is exceeded, then optimization iterations are stopped else step 3 is repeated until convergence. Maximum number of iterations taken up is 200 (Table 3).

The normalized weights $\left(W_{12}, W_{13}, W_{23}\right)$ have been calculated using equation 6 for QANN-P model by using PSO in the quadratic form (Table 4).

\section{Application of GA to Determine the Weight Coefficients}

In order to determine optimum weights using GA following steps were taken:

Step 1: Generate an initial population of random weights.

Step 2: ANN was evaluated using the population weights. This was done by computing the raining error and assigning it to a fitness value depending on the solutions.

Step 3: Parents for genetic manipulation were selected and a new population of weights were created. The best existing weights (reproduction) were copied. New weights were created by crossover and mutation operators.

Step 4: The best population of weights that appeared in any generation was designated as the result of the performed GA. The maximum number of generation was taken up as 100 which was used to stop the iteration (as shown in Table 2).

Table 4. Calculation of weights using PSO (Quadratic)

\begin{tabular}{|c|c|c|c|c|c|c|c|c|c|}
\hline Year & Per Capita & Consumer & Populatic & $\mathrm{X} 1^{\wedge} 2$ & $x 2^{\wedge} 2$ & $\times 3^{\wedge} 2$ & W12 & W13 & W 23 \\
\hline 2001 & 1.739234 & 2.11078 & 1.284397 & 3.002919 & 4.429865 & 1.643856 & 3.64415 & 2.701863 & 2.225637 \\
\hline 2002 & 1.740482 & 2.113684 & 1.285238 & 3.00315 & 4.438406 & 1.651702 & 3.645026 & 2.702687 & 2.230537 \\
\hline 2003 & 1.743535 & 2.118801 & 1.291731 & 3.002158 & 4.444737 & 1.660925 & 3.648868 & 2.705969 & 2.234403 \\
\hline 2004 & 1.742362 & 2.119748 & 1.299156 & 3.007993 & 4.44471 & 1.667298 & 3.652136 & 2.710261 & 2.236484 \\
\hline 2005 & 1.74656 & 2.122802 & 1.306171 & 3.014499 & 4.454473 & 1.670075 & 3.658678 & 2.716603 & 2.240316 \\
\hline 2006 & 1.757234 & 2.133471 & 1.307219 & 3.012913 & 4.461283 & 1.678114 & 3.670071 & 2.726663 & 2. 250227 \\
\hline 2007 & 1.765759 & 2.138782 & 1.32213 & 3.017055 & 4.468402 & 1.691601 & 3.679283 & 2.726751 & 2.255423 \\
\hline 2008 & 1.766098 & 2.135789 & 1.322586 & 3.014853 & 4.472879 & 1.69542 & 3.68148 & 2.735885 & 2.260517 \\
\hline 2009 & 1.765251 & 2.14489 & 1.328288 & 3.026726 & 4.484091 & 1.701831 & 3.690773 & 2.743303 & 2.263619 \\
\hline 2010 & 1.772931 & 2.145303 & 1.337613 & 3.027097 & 4.494202 & 1.707186 & 3.687869 & 2.745971 & 2. 268787 \\
\hline 2011 & 1.776363 & 2.152834 & 1.34153 & 3.028766 & 4.498124 & 1.71523 & 3.693322 & 2.748153 & 2.272937 \\
\hline 2012 & 1.781887 & 2.172139 & 1.33906 & 3.022918 & 4.498769 & 1.721589 & 3.702723 & 2.758801 & 2.277813 \\
\hline 2013 & 1.780678 & 2.177584 & 1.345109 & 3.030564 & 4.506537 & 1.723886 & 3.703262 & 2.762279 & 2.278135 \\
\hline 2014 & 1.758824 & 2.184461 & 1.354249 & 3.045545 & 4.524084 & 1.734389 & 3.69907 & 2.767402 & 2.272634 \\
\hline 2015 & 1.751147 & 2.213611 & 1.370402 & 3.065134 & 4.522264 & 1.732671 & 3.695373 & 2.761724 & 2.258565 \\
\hline
\end{tabular}


The weights $W_{1}, W_{2}, W_{3}$ are calculated as per Equation 7 for GA in linear form and as per Equation 8 for GA in quadratic form. The weights computed using GA in linear form are depicted in Table 5 where normalized demand (nDemand), normalized weights $\left(W_{1}, W_{2}\right.$ and $\left.W_{3}\right)$ and normalized values of per capita energy (nPercapita), consumer price index (nConPrIndx) and number of consumers(nCon) are fed as input to the ANN. The projected value of the energy demand (ANNGA) from the year 2001 to 2015 as obtained from ANN have been compared with the actual demand in Table 6.

With respect to ANN-GA model in quadratic form, Table 7 depicts the normalized weights $\left(W_{1}, W_{2}\right.$ and $\left.W_{3}\right)$ and normalized forecasted value of electricity demand (n-GA-Q). The forecasted value of electricity demand (ANN-GA-Q) have been compared with actual demand (nDemand) from the years 2001 to 2015 in Table 8 .

\section{APPLICATION AND DISCUSSION}

The proposed ANN-PSO and ANN-GA models in linear and quadratic forms have been implemented in SCILAB computing environment which gives easy access to compare with other forecasting methods. The demand data in the sectors of agriculture, residential, commercial, industries along with total electric energy demand from the years 1991 to 2015 have been used for the proposed hybrid ANN models using PSO and GA techniques. Forecasts for each sector by each model have been mentioned in Table 9, Table 10, Table 11, Table 12 and Table 13.

Table 5. Computation of weights using GA

\begin{tabular}{|c|c|c|c|c|c|c|c|}
\hline Year & nPerCapita & nConPrIndx & nCon & nDemand & w1 & w2 & w3 \\
\hline 2001 & 1.82 & 2.14 & 1.33 & 0.2298 & -1.9 & 1.45 & 0.84 \\
\hline 2002 & 2.4 & 2.22 & 1.35 & 0.2591 & -1.7 & 1.81 & 0.67 \\
\hline 2003 & 2.51 & 2.27 & 1.35 & 0.3004 & -1.76 & 1.82 & 0.67 \\
\hline 2004 & 2.64 & 2.29 & 1.37 & 0.3271 & -1.77 & 1.81 & 0.67 \\
\hline 2005 & 2.91 & 2.39 & 1.42 & 0.3626 & -1.76 & 1.95 & 0.76 \\
\hline 2006 & 3.25 & 2.43 & 1.45 & 0.3858 & -1.72 & 2 & 0.76 \\
\hline 2007 & 3.39 & 2.58 & 1.48 & 0.4023 & -1.72 & 2 & 0.76 \\
\hline 2008 & 3.39 & 2.83 & 1.51 & 0.4399 & -1.72 & 2 & 0.76 \\
\hline 2009 & 3.66 & 3.14 & 1.54 & 0.523 & -1.7 & 2 & 0.76 \\
\hline 2010 & 3.52 & 3.45 & 1.59 & 0.5296 & -1.62 & 1.7 & 0.32 \\
\hline 2011 & 3.64 & 3.39 & 1.58 & 0.5481 & -1.62 & 1.7 & 0.32 \\
\hline 2012 & 3.79 & 3.31 & 1.55 & 0.5258 & -1.64 & 1.57 & 0.77 \\
\hline 2013 & 3.93 & 3.28 & 1.54 & 0.5463 & -1.61 & 1.03 & 1.11 \\
\hline 2014 & 4.07 & 2.99 & 1.51 & 0.5777 & -1.7 & 1.77 & 1.11 \\
\hline 2015 & 4.27 & 2.89 & 1.54 & 0.6026 & -1.67 & 1.92 & 1.5 \\
\hline
\end{tabular}


Table 6. Forecasting of demand using ANN-GA

\begin{tabular}{|r|r|r|r|r|r|}
\hline Year & PerCapita & ConPricel| Consumer & Demand & ANN-GA \\
\hline 2001 & 539 & 103 & 1648341 & 9095 & 9084.51 \\
\hline 2002 & 708 & 107 & 1675165 & 9412 & 9393.63 \\
\hline 2003 & 740 & 109 & 1676113 & 9030 & 9050.17 \\
\hline 2004 & 780 & 110 & 1702541 & 9382 & 9359.28 \\
\hline 2005 & 860 & 115 & 1768052 & 9919 & 9925.99 \\
\hline 2006 & 960 & 117 & 1801972 & 10349 & 10320.97 \\
\hline 2007 & 1000 & 124 & 1839241 & 10912 & 10870.51 \\
\hline 2008 & 1000 & 136 & 1872734 & 10520 & 10509.88 \\
\hline 2009 & 1080 & 151 & 1913697 & 11940 & 11918.06 \\
\hline 2010 & 1040 & 166 & 1972563 & 12625 & 12639.33 \\
\hline 2011 & 1073 & 163 & 1967024 & 10425 & 10389.67 \\
\hline 2012 & 1118 & 159 & 1932771 & 10085 & 10063.38 \\
\hline 2013 & 1161 & 157 & 1919531 & 10091 & 10046.21 \\
\hline 2014 & 1200 & 143 & 1874940 & 12301 & 12313.04 \\
\hline 2015 & 1259 & 138 & 1909856 & 12500 & 12501.94 \\
\hline
\end{tabular}

Table 7. Computation of weights using GA (Quadratic)

\begin{tabular}{|r|r|r|r|r|r|r|r|r|}
\hline 2001 & 539 & 103 & 1648341 & -0.0015 & 0.001369 & 0.00209 & 0.52961 & 0.5289 \\
\hline 2002 & 708 & 107 & 1675165 & -0.00157 & 0.00226 & 0.0023 & 0.54807 & 0.5479 \\
\hline 2003 & 740 & 109 & 1676113 & -0.0036 & 0.0031 & 0.0069 & 0.525825 & 0.5248 \\
\hline 2004 & 780 & 110 & 1702541 & -0.0035 & 0.00769 & 0.0018 & 0.546323 & 0.5458 \\
\hline 2005 & 860 & 115 & 1768052 & -0.0025 & 0.0026 & 0.0053 & 0.577593 & 0.577 \\
\hline 2006 & 960 & 117 & 1801972 & -0.00346 & 0.002237 & 0.0045 & 0.602632 & 0.601 \\
\hline 2007 & 1000 & 124 & 1839241 & -0.00226 & 0.0014 & 0.00716 & 0.635416 & 0.6349 \\
\hline 2008 & 1000 & 136 & 1872734 & -0.0036 & 0.0062 & 0.0088 & 0.61259 & 0.611 \\
\hline 2009 & 1080 & 151 & 1913697 & -0.009 & 0.0069 & 0.033 & 0.695277 & 0.6923 \\
\hline 2010 & 1040 & 166 & 1972563 & -0.0093 & 0.008321 & 0.0025 & 0.735166 & 0.7345 \\
\hline 2011 & 1073 & 163 & 1967024 & -0.0057 & 0.00469 & 0.00482 & 0.607058 & 0.6068 \\
\hline 2012 & 1118 & 159 & 1932771 & -0.00215 & 0.0055 & 0.0034 & 0.587259 & 0.5868 \\
\hline 2013 & 1161 & 157 & 1919531 & -0.00437 & 0.0024 & 0.004344 & 0.587608 & 0.5874 \\
\hline 2014 & 1200 & 143 & 1874940 & -0.0042 & 0.006047 & 0.00613 & 0.716299 & 0.716 \\
\hline 2015 & 1259 & 138 & 1909856 & -0.00191 & 0.000219 & 0.00531 & 0.727887 & 0.7269 \\
\hline
\end{tabular}


Table 8. Calculation of demand by QANN-GA

\begin{tabular}{|r|r|r|r|r|r|r|}
\hline 2001 & 539 & 103 & 1648341 & 9095 & 0.5302 & 9105.125 \\
\hline 2002 & 708 & 107 & 1675165 & 9412 & 0.549 & 9427.977 \\
\hline 2003 & 740 & 109 & 1676113 & 9030 & 0.5235 & 8990.066 \\
\hline 2004 & 780 & 110 & 1702541 & 9382 & 0.5467 & 9388.479 \\
\hline 2005 & 860 & 115 & 1768052 & 9919 & 0.5756 & 9884.779 \\
\hline 2006 & 960 & 117 & 1801972 & 10349 & 0.6019 & 10336.43 \\
\hline 2007 & 1000 & 124 & 1839241 & 10912 & 0.633 & 10870.51 \\
\hline 2008 & 1000 & 136 & 1872734 & 10520 & 0.6103 & 10480.68 \\
\hline 2009 & 1080 & 151 & 1913697 & 11940 & 0.6962 & 11955.84 \\
\hline 2010 & 1040 & 166 & 1972563 & 12625 & 0.7347 & 12617 \\
\hline 2011 & 1073 & 163 & 1967024 & 10425 & 0.6078 & 10437.75 \\
\hline 2012 & 1118 & 159 & 1932771 & 10085 & 0.5849 & 10044.49 \\
\hline 2013 & 1161 & 157 & 1919531 & 10091 & 0.5887 & 10109.75 \\
\hline 2014 & 1200 & 143 & 1874940 & 12301 & 0.714 & 12261.52 \\
\hline 2015 & 1259 & 138 & 1909856 & 12500 & 0.7274 & 12491.64 \\
\hline
\end{tabular}

Table 9. Agriculture sector electricity demand forecast

\begin{tabular}{|r|r|r|r|r|r|r|r|r|r|}
\hline Year & Actual & ANN-PSO QANN-P & ANN-GA & QANN-G & ARIMA & Linear & ANN-BP \\
\hline 2001 & 9095 & 9119 & 9102 & 9084 & 9105 & 9114 & 8635 & 9067 \\
\hline 2002 & 9412 & 9411 & 9428 & 9394 & 9428 & 9050 & 9195 & 9435 \\
\hline 2003 & 9030 & 9033 & 9016 & 9050 & 8990 & 9217 & 9223 & 9046 \\
\hline 2004 & 9382 & 9411 & 9411 & 9359 & 9388 & 9321 & 9551 & 9377 \\
\hline 2005 & 9919 & 9926 & 9926 & 9926 & 9885 & 10205 & 10240 & 9899 \\
\hline 2006 & 10349 & 10338 & 10372 & 10321 & 10336 & 10497 & 10760 & 10344 \\
\hline 2007 & 10912 & 10905 & 10939 & 10870 & 10870 & 10896 & 11011 & 10938 \\
\hline 2008 & 10520 & 10527 & 10527 & 10510 & 10481 & 11094 & 10973 & 10499 \\
\hline 2009 & 11940 & 11935 & 11952 & 11918 & 11956 & 11063 & 11116 & 11933 \\
\hline 2010 & 12625 & 12639 & 12656 & 12639 & 12617 & 12027 & 11137 & 12648 \\
\hline 2011 & 10425 & 10424 & 10441 & 10390 & 10438 & 11877 & 11257 & 10429 \\
\hline 2012 & 10085 & 10080 & 10115 & 10063 & 10044 & 10773 & 11154 & 10081 \\
\hline 2013 & 10091 & 10115 & 10132 & 10046 & 10110 & 11037 & 11179 & 10112 \\
\hline 2014 & 12301 & 12279 & 12313 & 12313 & 12261 & 10680 & 11261 & 12301 \\
\hline 2015 & 12500 & 12536 & 12468 & 12502 & 12491 & 12289 & 11895 & 12524 \\
\hline
\end{tabular}


Table 10. Residential sector electric demand forecast

\begin{tabular}{|r|r|r|r|r|r|r|r|r|}
\hline Year & QANN-G & ANN-GA & ACTUAL & QANN-P & ARIMA & ANN-PSO Linear & ANN-BP \\
\hline 2001 & 174 & 171 & 173 & 175 & 201 & 175 & 134 & 172 \\
\hline 2002 & 184 & 178 & 180 & 180 & 180 & 177 & 194 & 180 \\
\hline 2003 & 183 & 195 & 190 & 191 & 147 & 189 & 189 & 190 \\
\hline 2004 & 184 & 187 & 185 & 182 & 166 & 185 & 216 & 183 \\
\hline 2005 & 196 & 190 & 190 & 187 & 185 & 190 & 221 & 190 \\
\hline 2006 & 194 & 194 & 194 & 199 & 230 & 192 & 213 & 195 \\
\hline 2007 & 219 & 219 & 216 & 211 & 216 & 217 & 247 & 216 \\
\hline 2008 & 236 & 231 & 237 & 237 & 265 & 235 & 276 & 239 \\
\hline 2009 & 400 & 402 & 403 & 402 & 374 & 404 & 309 & 400 \\
\hline 2010 & 494 & 495 & 498 & 498 & 510 & 502 & 415 & 402 \\
\hline 2011 & 506 & 511 & 509 & 510 & 512 & 511 & 494 & 329 \\
\hline 2012 & 520 & 518 & 518 & 519 & 514 & 517 & 580 & 319 \\
\hline
\end{tabular}

Table 11. Commercial sector electric demand forecast

\begin{tabular}{|r|r|r|r|r|r|r|r|r|r|}
\hline Year & ANN-BP & Actual & ANN-GA & ANN-PSO Q-ANN-G & Q-ANN-P & Linear & ARIMA \\
\hline 2001 & 974.97 & 978 & 979 & 970 & 979 & 980 & 1051 & 1054 \\
\hline 2002 & 1032.02 & 1038 & 1039 & 1032 & 1032 & 1044 & 1070 & 1098 \\
\hline 2003 & 1081.27 & 1080 & 1092 & 1096 & 1087 & 1070 & 1050 & 1087 \\
\hline 2004 & 1097.65 & 1103 & 1104 & 1094 & 1102 & 1106 & 1087 & 1104 \\
\hline 2005 & 1184.28 & 1179 & 1181 & 1175 & 1175 & 1178 & 1128 & 1136 \\
\hline 2006 & 1217.75 & 1216 & 1217 & 1214 & 1216 & 1216 & 1178 & 1159 \\
\hline 2007 & 1323.26 & 1331 & 1331 & 1338 & 1329 & 1329 & 1234 & 1204 \\
\hline 2008 & 1337 & 1353 & 1363 & 1348 & 1358 & 1355 & 1303 & 1221 \\
\hline 2009 & 1037.18 & 1043 & 1039 & 1037 & 1053 & 1044 & 1391 & 1290 \\
\hline 2010 & 1589.04 & 1592 & 1599 & 1588 & 1590 & 1594 & 1499 & 1614 \\
\hline 2011 & 1618.18 & 1611 & 1611 & 1621 & 1604 & 1609 & 1577 & 1629 \\
\hline 2012 & 1725.58 & 1711 & 1705 & 1712 & 1705 & 1707 & 1636 & 1665 \\
\hline 2013 & 1914.53 & 1917 & 1920 & 1935 & 1934 & 1915 & 1943 & 1925 \\
\hline & & & & & & & & \\
\hline
\end{tabular}

Table 12. Industries sector electricity demand forecast

\begin{tabular}{|r|r|r|r|r|r|r|r|r|}
\hline Year & Actual & ANN-PSO & Q-ANN-P & ANN-GA & Q-ANN-G Linear & ANN-BP & ARIMA \\
\hline 2001 & 12085 & 12002 & 12143 & 12186 & 12114 & 10960 & 12025 & 11552 \\
\hline 2002 & 12588 & 12528 & 12758 & 12607 & 12459 & 13364 & 12639 & 13894 \\
\hline 2003 & 14168 & 14017 & 14004 & 14047 & 17127 & 17127 & 14323 & 16603 \\
\hline 2004 & 14547 & 14568 & 14456 & 14499 & 14554 & 17240 & 14535 & 15943 \\
\hline 2005 & 18001 & 17985 & 17947 & 17958 & 17959 & 18107 & 17929 & 17114 \\
\hline 2006 & 20977 & 21018 & 20946 & 20857 & 20999 & 19010 & 21069 & 19083 \\
\hline 2007 & 22948 & 23038 & 22825 & 22900 & 22888 & 19766 & 22837 & 20576 \\
\hline 2008 & 23115 & 23137 & 23139 & 23319 & 23128 & 20867 & 23358 & 22164 \\
\hline 2009 & 24849 & 24846 & 24667 & 24765 & 24815 & 22823 & 24950 & 23481 \\
\hline 2010 & 25107 & 25093 & 25152 & 25225 & 24909 & 23711 & 24999 & 24674 \\
\hline 2011 & 18186 & 18214 & 18183 & 18164 & 18119 & 23113 & 18142 & 23578 \\
\hline 2012 & 21105 & 21021 & 21130 & 21080 & 21121 & 21792 & 21004 & 19338 \\
\hline 2013 & 15605 & 15693 & 15655 & 15401 & 15619 & 15038 & 15407 & 15879 \\
\hline 2014 & 15387 & 15444 & 15310 & 15442 & 15421 & 14654 & 15246 & 14696 \\
\hline 2015 & 17072 & 16963 & 17182 & 17188 & 17029 & 18167 & 17138 & 17438 \\
\hline
\end{tabular}


Table 13. Total electricity demand forecast

\begin{tabular}{|r|r|r|r|r|r|r|r|r|}
\hline S.No & Year & Actual & ANN-PSO ANN-GA & QANN-G & QANN-P & ARIMA & Linear \\
\hline 1 & 2001 & 36577 & 36456 & 35342 & 34483 & 36630 & 41525 & 37643 \\
\hline 2 & 2002 & 38529 & 38041 & 57598 & 42589 & 38296 & 39275 & 40247 \\
\hline 3 & 2003 & 46130 & 46323 & 35857 & 27580 & 46161 & 41540 & 42787 \\
\hline 4 & 2004 & 49712 & 49898 & 72298 & 16331 & 49990 & 46339 & 45829 \\
\hline 5 & 2005 & 51282 & 51678 & 30070 & 7127 & 51210 & 49769 & 48925 \\
\hline 6 & 2006 & 49485 & 49478 & 28284 & 33230 & 49853 & 52421 & 51870 \\
\hline 7 & 2007 & 56493 & 56725 & 45852 & 59521 & 56585 & 53798 & 54343 \\
\hline 8 & 2008 & 53506 & 53167 & 44152 & 41971 & 53837 & 57398 & 57267 \\
\hline 9 & 2009 & 57212 & 57113 & 48771 & 46144 & 57564 & 58432 & 59603 \\
\hline 10 & 2010 & 60518 & 60585 & 40133 & 26584 & 60689 & 60480 & 62076 \\
\hline 11 & 2011 & 61897 & 62044 & 61170 & 49578 & 62080 & 63373 & 64631 \\
\hline 12 & 2012 & 66391 & 66274 & 48170 & 51588 & 66459 & 66397 & 67069 \\
\hline 13 & 2013 & 72987 & 73224 & 36304 & 28095 & 73260 & 70177 & 69712 \\
\hline 14 & 2014 & 74990 & 74704 & 45852 & 47724 & 74685 & 74547 & 72748 \\
\hline 15 & 2015 & 77218 & 77181 & 73947 & 53408 & 77690 & 78158 & 75681 \\
\hline
\end{tabular}

\section{Accuracy Measures}

The mean absolute percentage error (MAPE) and root mean square error (RMSE) measure the residual errors, which give a global idea of the difference between the predicted and actual values. In the present study, the RMSE, MAPE, have been used for comparison and have been defined as follows:

$$
\begin{aligned}
& M A P E=\Sigma\left[\frac{\frac{Y_{\text {actual }}-Y_{\text {estimated }}}{Y_{\text {actual }}}}{n}\right] * 100 \\
& R M S E=\sqrt{\frac{\sum \frac{\left(Y_{\text {actual }}-Y_{\text {estimated }}\right)^{2}}{n}}{n}}
\end{aligned}
$$

where $Y_{\text {actual }}, Y_{\text {estimated }}$ are the actual and estimated values and $n$ is the number of observations.

\section{Forecasts Comparisons}

The results of ANN-PSO model in both linear and quadratic forms have been compared with ARIMA and linear models using RMSE and MAPE (Table 14). The hybrid ANN models (with PSO and GA optimisation) consistently outperform the ARIMA and linear models in all the sectors. ANN-PSO and ANN-GA models have better performance than ANN-BP which indicates that optimisation by PSO and GA improves the performance of ANN-BP. Amongst the four hybrid ANN models (ANNPSO, ANN-GA, QANN-P and QANN-G), ANN-PSO is the best fit model in all the sectors based on RMSE and MAPE. 
Table 14. Comparison of forecasts of various models for different sectors

\begin{tabular}{|r|l|r|r|r|r|r|r|r|r|}
\hline S.no & Model & \multicolumn{9}{|c|}{ AgricultureDemand } & \multicolumn{7}{|c|}{ Residential Demand CommercialDemand Industries Demand } \\
\hline & & RMSE & MAPE & RMSE & MAPE & RMSE & MAPE & RMSE & MAPE \\
\hline 1 & ANN-PSO & 16.95 & 0.12 & 1.94 & 0.17 & 59 & 0.29 & 59 & 0.29 \\
\hline 2 & ANN-GA & 23.9 & 0.2 & 2.35 & 0.85 & 109.7 & 0.52 & 109.7 & 0.52 \\
\hline 3 & QANN-P & 22.95 & 0.19 & 2.49 & 0.86 & 96.3 & 0.47 & 96.3 & 0.47 \\
\hline 4 & QANN-G & 26.69 & 0.22 & 3.43 & 1.17 & 75.75 & 0.32 & 75.75 & 0.32 \\
\hline 5 & ANN-BP & 61.39 & 0.15 & 162.24 & 40.3 & 119.2 & 0.57 & 119.2 & 0.57 \\
\hline 6 & ARIMA & 724.7 & 4.92 & 22.56 & 17.25 & 1927 & 8.2 & 1927 & 8.2 \\
\hline 7 & LINEAR & 738 & 617 & 46.83 & 13.34 & 2157 & 9.8 & 2157 & 9.8 \\
\hline
\end{tabular}

Forecasting comparison of the total electricity demand is listed in Table 15. It is evident that QANN-P model outperforms other models in terms of RMSE and MAPE values. From Figure 2 it is evident that QANN-P aligns with the actual demand over the years 2001 to 2015.

Hence QANN-P is used to forecast the electricity demand for the years beyond 2015.

Table 16 lists the forecasts from the years 2001 to 2020 using QANN-P model and Figure 3 shows the profile of the projected demand using QANN-P model against the actual demand from the years 2001 till the year 2020 .

\section{CONCLUSION}

ANN-PSO both in linear and quadratic form gives better results when compared with the ANN -BP or time series model such as ARIMA. As is seen in the five-time series the forecasting ability of ANN improves when optimized with PSO. Hence for power utilities in developing economies such as Tamil Nadu Generation and Distribution Company (TANGEDCO) both linear and quadratic versions of ANN-PSO models are ideal for forecasting electricity demand. For optimizing ANN, PSO performs better than GA hence PSO can be the better optimizing technique. For further studies a combined optimizing technique based on PSO and GA can be considered.

Table 15. Comparison of forecasts of total electricity demand

\begin{tabular}{|l|r|r|r|r|r|r|r|}
\hline Error & ANN-BP & ANN-GA & QANN-G & ANN-PSO & QANN-P & ARIMA & Linear \\
\hline RMSE & 258.16 & 47.63 & 6256 & 236 & 65 & 2626 & 9398 \\
\hline MAE & 198 & 15496 & 20,000 & 196 & 218.9 & 2108 & 2338 \\
\hline MAPE & 0.72 & 27 & 34 & 0.37 & 0.38 & 4.22 & 4.28 \\
\hline
\end{tabular}


Table 16. Forecasting electricity demand by QANN-P model

\begin{tabular}{|r|r|r|r|r|}
\hline Year & P-Cap-En & Co-Pr-Ind & N-Cons & QANN-P \\
\hline 2001 & 554.6318 & 472.6592 & 1616811 & 36630 \\
\hline 2002 & 586.9038 & 514.69 & 1637313 & 38296 \\
\hline 2003 & 604.9707 & 527.9458 & 1657332 & 46161 \\
\hline 2004 & 632.8892 & 550.1697 & 1699559 & 49990 \\
\hline 2005 & 680.9822 & 598.7729 & 1703774 & 51210 \\
\hline 2006 & 709.9936 & 644.1522 & 1726785 & 49853 \\
\hline 2007 & 754.7332 & 658.1305 & 1764567 & 56585 \\
\hline 2008 & 778.6538 & 680.9808 & 1766763 & 53837 \\
\hline 2009 & 793.0188 & 716.231 & 1795486 & 57564 \\
\hline 2010 & 823.5501 & 749.5662 & 1817832 & 60689 \\
\hline 2011 & 831.9718 & 759.9379 & 1853117 & 62080 \\
\hline 2012 & 856.7116 & 779.4751 & 1878436 & 66459 \\
\hline 2013 & 901.9753 & 814.7637 & 1905657 & 73260 \\
\hline 2014 & 922.002 & 835.0401 & 1923826 & 74685 \\
\hline 2015 & 930.8125 & 851.1199 & 1966485 & 77690 \\
\hline 2016 & 949.2263 & 859.4808 & 1995669 & 74204 \\
\hline 2017 & 981.104 & 884.6208 & 2044049 & 76370 \\
\hline 2018 & 1009.227 & 914.1552 & 2093418 & 78862 \\
\hline 2019 & 1056.216 & 948.3732 & 2129758 & 81538 \\
\hline 2020 & 1074.459 & 961.182 & 2159453 & 84714 \\
\hline
\end{tabular}

Figure 2. Total demand by ANN-PSO models

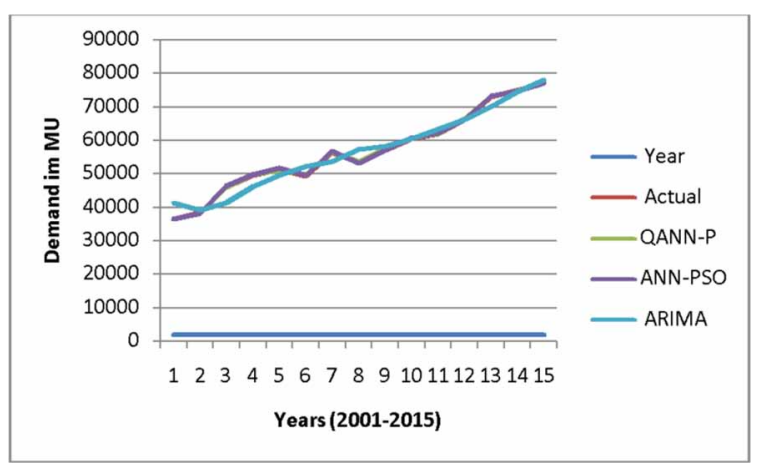




\section{Figure 3. Comparison of QANN-PSO forecast with actual demand}

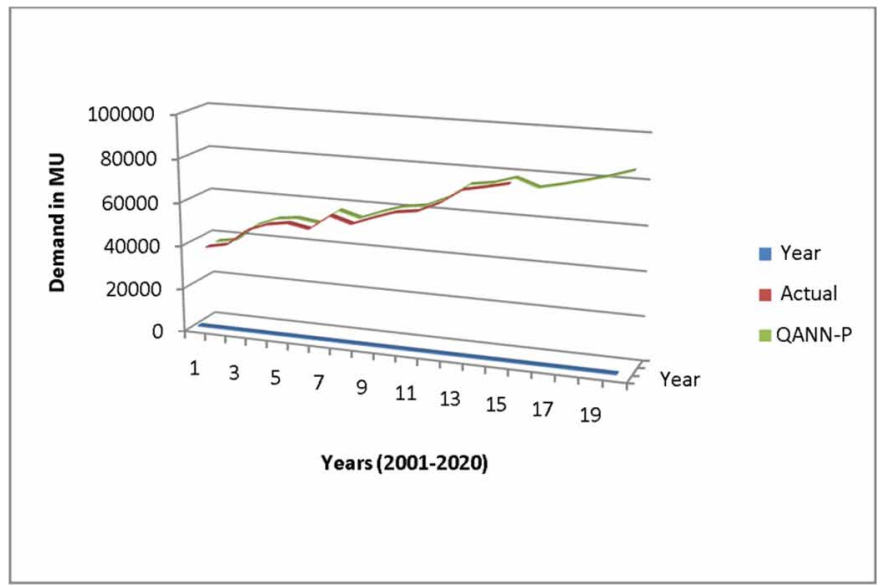




\section{REFERENCES}

Yu, S. W., \& Zhu, K. J. (2011). A hybrid procedure for energy demand forecasting in China. Elsevier.

Adhikari, R., Agrawal, R.K., \& Laxmi, K. (2013). PSO based Neural Networks vs Traditional Statistical Models for seasonal Time Series Forecasting.

Assareh, E., Behrang, M. A., Assareh, R., \& Hedayat, N. (2011). Global Electricity Consumption Estimation Using Particle Swarm Optimisation. PSO.

Banos, R., Manzano-Agugliaro, F., Montoya, F. G., Gil, C., Alcayde, A., \& Gómez, J. (2011). Optimization methods applied to renewable and sustainable energy: A review. Renewable \& Sustainable Energy Reviews, 15(4), 1753-1766.

Chang, C.-Y. F., \& Lin, J.-J. (2010). Monthly electricity demand forecasting based on a weighted evolving fuzzy neural network approach. Elsevier.

Codoni, R., Park, H. C., \& Ramani, K. V. (1985). Integrated Energy Planning - A Manual. Kuala Lumpur: Asia Pacific Development Centre.

El, A., \& Yorino, N. (2010). A hybrid PSO technique for procuring VAR ancillary service in the deregulated electricity markets. Electrical Power and Energy Systems, 32(6), 664-670. doi:10.1016/j.ijepes.2009.11.025

Engelbrecht, A. P., Cloete, I., Geldenhuys, J., \& Zurada, J. M. (1996). Automatic scaling using gamma learning for feed forward neural networks. Proceedings of the International Workshop on Artificial Neural Networks: From Natural to Artificial Neural Computation.

Feinberg, E.A., \& Genethlion, D. (2006). Load Forecasting. In Applied Mathematics for Power System weather (Ch. 12, pp. 269 -285).

Government of India. (2006). Integrated Energy Policy - Report of the Expert Committee, Planning Commission. Retrieved from http://planningcommission.nic.in/reports/genrep/rep_intengy.pdf

Granger, C. W. J., \& Terasvirta, T. (1993). Modelling Nonlinear Econ-Grover, R.B. and S. Chandra, 2006, Scenario for growth of electricity in India. Energy Policy, 34, 2834-2847.

Harvey, A. C. (1997). Trends, cycles and autoregressions. The Economic Journal, 107(440), 192-201. doi:10.1111/1468-0297.00152

Hunt, L.C., Judge, G., \& Ninomiya, Y. (2003). Underlying trends and seasonality in UK energy demand: A sectoral analysis. Energy Economics, 25(1), 93-118. doi:10.1016/S0140-9883(02)00072-5

Central Electricity Authority India. (2010). Tamil Nadu Electricity Board Tariff Order 2010.

Kaastra, I., \& Boyd, M. (1995). Designing a neural network for forecasting financial and economic time series. Neurocomputing.

Kodeeswararamanathan, M., Stalin, N., Ramasubramanian, S., \& Krishnamoorthy, R. (n. d.). Mathematical modelling approach for solutions in power consumption issues of Tamil Nadu. Journal of Chemical and Pharmaceutical Sciences.

Nazari, H., Kazemi, A., \& Nazari, M. (2015). Evaluating the performance of genetic and Particle Swarm Optimisation algorithms to select an appropriate scenario for forecasting energy demand using economic indicators: Residential and Commercial Sectors in Iran. Int. J. Energy Environ. Engg., 6(4), 345-355.

Pany, P. K., \& Ghoshal, S. P. (2013). Day-ahead Electricity Price Forecasting Using PSO-Based LLWNN Model. International Journal of Energy Engineering, 3(4), 99-106.

Bhattacharyya, S.C., \& Timilsina, G.R. (2009). Energy Demand Models for Policy Formulation, A Comparative Study of Energy Demand Models.

Singh, A., Dixit, S., \& Srivastava, L. (2011). Particle Swarm Optimization- Artificial Neural Network For Power System Load Flow. International Journal of Power System Operation \& Energy Management, 1(2), 73-82. 
Trippi, R.R., \& Turban, E. (1992). Neural Networks in Finance and Investing: Using Artificial Intelligence to improve real world performance.

Unler, A. (2008). Improvement of Energy demand forecasts using Swarm intelligence: The case of Turkey with projections to 2025. Energy Policy, 36(6), 1937-1944.

Westoby, R., \& Pearce, D. (1984). Single equation models for the projection of energy demand in the United Kingdom, 195480. Scottish Journal of Political Economy, 31(3), 229-254. doi:10.1111/j.1467-9485.1984. tb00476.x

Yu, S., Wei, Y.-M., \& Wang, K. (2012). A PSO-GA optimal model to estimate primary energy demand of China. Journal of Energy Policy, 42(C), 329-340. doi:10.1016/j.enpol.2011.11.090

Zhang, G., Patuwo, B.E., \& Hu, M.Y. (1998). Forecasting with artificial neural networks: The state of the art. International Journal of Forecasting, 14, 35-62. doi:10.1016/S0169-2070(97)00044-7

Zhu, S., Wang, J., Zhao, W., \& Wang, J. (2011). A seasonal hybrid procedure for electricity demand forecasting in China. Applied Energy, 88(11), 3807-3815. doi:10.1016/j.apenergy.2011.05.005 